\title{
FORUM
}

\section{GMT and Longitude by Lunar Distance: Two Methods Compared From a Practitioner's Point of View}

\author{
Eric Romelczyk \\ (E-mail: eric.romelczyk@gmail.com)
}

\begin{abstract}
This article discusses the technique of observing lunar distance - that is, angular distance between the moon and another celestial body - to establish universal time and longitude, from a practitioner's point of view. The article presents a brief overview of the principles underlying the lunar distance observation and its use in celestial navigation. A discussion follows of two different methods for finding universal time by observing lunar distance, Dr. Wendel Brunner's calculator-based method and the specialised inspection tables created by Bruce Stark. The article compares the two methods against each other for ease of use and accuracy. The author concludes that either method will provide satisfactory results, but that the technique of observing lunar distance is unlikely to regain relevance in the modern-day practice of navigation and is primarily useful as a skill-building exercise in making sextant observations.
\end{abstract}

\section{KE Y W OR D S}

$\begin{array}{lll}\text { 1. Navigation. } & \text { 2. History. } & \text { 3. Nautical. Time. }\end{array}$

Submitted: 8 August 2018. Accepted: 14 April 2019. First published online: 2 May 2019.

\section{INTRODUCTION.}

1.1. History of the lunar distance method. For centuries of seafaring history, a method for accurately measuring time to the degree of precision necessary to establish the navigator's longitude was out of reach for practical purposes. It had been understood since the mid-16th century that the navigator's longitude could be established either by reference to the moon's angular distance from other celestial bodies - the "lunar distance", measured by careful sextant observations - or by reference to a timepiece of sufficient accuracy. Initially, the cost of the chronometer kept it largely out of reach of the average mariner. As for the lunar distance method, the mathematics involved in determining time and longitude by lunar distance remained beyond the grasp of most seafarers until Maskelyne's (1767) publication of a "Nautical Almanac" and its associated tables, and Bowditch's publication of a simplified method in the "New American Practical Navigator" of 1802 (National Geo-spatial Intelligence Agency, 2017a; 2017b).

By the late nineteenth century, the increasingly affordable chronometer had established sufficient primacy for Lecky and Allingham (1918. P462), in their famous work, to declare the lunar distance method "deader than Julius Caesar". However, interest in the lunar distance method has never waned completely despite the technique having long since faded 
from the standard repertoire of operations in celestial navigation. Below the author presents a discussion of two modern methods of determining time and longitude by lunar distance.

1.2. Contemporary practice of lunar distance observations. Starpath Navigation LLC continues to publish a set of specialised logarithm tables by Stark (2010), now in its third edition, for establishing universal time from observed lunar distance. This publication presents the process in a concise tabular format. In Stark's tables, the trigonometric functions of the angular distances involved are rendered as logarithms and solutions are found by addition and subtraction.

Attacking the problem from a slightly different angle is the iterative, calculator-based method published on the Starpath Navigation website by Brunner (2005). This method uses the formulae of spherical trigonometry for solving the typical celestial triangle, substituting values so as to use the calculated altitude of the moon, the calculated altitude of the other body, and the difference in azimuths between the two bodies instead of, respectively, declination of the body, latitude of the observer, and Local Hour Angle (LHA) of the body as in the more familiar celestial triangle. Those conversant with sight reduction by calculator-based methods will find Brunner's method familiar.

Following the usage of both Brunner and Stark, the terms "Universal Time (UT)" and "Greenwich Mean Time (GMT)" are used interchangeably to refer to mean solar time at $0^{\circ}$ longitude.

Sadler (1978) deals with - and largely dismisses as unsuitable - a different method of determining longitude by successive lunar altitudes, as proposed by Sir Francis Chichester.

\section{METHODS.}

2.1. Principle of the lunar distance observation. Determination of time and therefore observer's longitude by lunar distance is possible because the difference in apparent motion between the moon and other bodies causes the angular distances between the moon and these bodies to change measurably from one hour to the next. Comparison of the observed distance with calculated distances for known intervals of GMT can thus be used to establish GMT at the time of observation. In the heyday of the lunar distance method, such tabulated distances, called "comparing distances," were included at three-hour intervals for various prominent bodies in the Nautical Almanac (Raper, 1908).

The difference in apparent motion between the moon and stars is a result primarily of the moon's change in Right Ascension (RA) relative to the stars and secondarily of the moon's change in declination relative to those bodies. As the moon completes one complete orbit in 27.3 days, it must move relative to the stars at a rate of $13.2^{\circ}$ per day, or approximately 33 arcminutes per hour. The net apparent motion of the moon averages one diameter, or approximately $0 \cdot 5^{\circ}$ of arc, per hour of GMT.

This difference of apparent motion makes the method demanding to use in a practical context. The moon's apparent motion amounting to approximately 30 arcseconds per minute of time, a measurement error of 1 arcminute will result in a time error of 2 minutes of time, or $0.5^{\circ}$ of longitude. Even a measurement error of 0.1 arcminute, essentially negligible in most celestial position-fixing, will translate to an error of 12 seconds of time, or up to 3 nautical miles position error, depending on latitude. Under anything less than perfect conditions at sea, limiting the measurement error to less than 1 arcminute can be very challenging. 
2.2. Observing lunar distance. In observing lunar distances, it is critical to take multiple distances, as carefully as possible, and average them to reduce random error. Brunner (2005) recommends plotting the observations carefully on graph paper, discarding obvious outliers, then drawing a best-fit linear regression line through the remaining points. Again, it must be remembered that in this operation, very small errors in observation translate to significant inaccuracy in the final result. A stable platform for the observer is critical in order to bring the limbs of both bodies into accurate alignment. Vessel motion, vibration and similar factors must be minimised as much as possible.

2.3. Finding GMT using Stark's tables. The first step in using Stark's tables is to "clear" the observed lunar distance. To "clear" a lunar distance is to correct it for the effects of parallax, refraction, semi-diameter and augmentation, as well as for Index Error (IE) of the sextant, so as to arrive at the true, geocentric lunar distance observed. In order to use Stark's tables, the observer must take altitudes of the Moon and of the other body, both before and after observing the lunar distance itself, or otherwise have altitudes taken of both bodies simultaneously with the lunar distance observation. This provides the necessary entering arguments for the first work form (Stark, 2010. P. x - xiii).

The observer must then compute comparing distances for the whole hour preceding the observation and that following the observation using Stark's Tables (Stark, 2010. P. xiv).

The cleared lunar distance is compared to the computed distances for the GMT hour preceding and following the observation. The difference between the calculated lunar distance for the preceding whole hour of GMT and the observed, cleared lunar distance is compared to the difference in lunar distance between the two whole hours. This difference is then converted to time by reference to Stark's Table 8. This gives the GMT increment in minutes and seconds to be added to the whole-hour GMT corresponding to the first calculated distance.

2.4. Finding time and longitude by Brunner's method. In the Brunner method, the navigator starts with an assumed time, an assumed longitude based on the assumed time, and a known latitude. The starting assumed time and assumed longitude need not be particularly close to the truth; as each iteration is worked through, the assumed time and assumed longitude is corrected until the lunar distance so calculated agrees with the lunar distance actually observed.

In this process, the navigator begins by calculating the true lunar distance for the assumed time and position using the formulae:

$$
\begin{aligned}
\operatorname{Sin} H C & =(\sin \mathrm{D} \sin \mathrm{L})+(\cos \mathrm{D} \cos \mathrm{L} \cos \mathrm{LHA}) \\
\cos \mathrm{Z}= & (\sin \mathrm{D}-(\sin \mathrm{HC} \sin \mathrm{L})) /(\cos \mathrm{HC} \cos \mathrm{L}) \\
\cos \mathrm{LD}= & (\sin (\mathrm{HO} \text { Body }) \sin (\mathrm{HO} \text { Moon })) \\
& +(\cos (\text { HO Body }) \cos (\text { HO Moon }) \cos (\mathrm{Z} \text { Body }-\mathrm{Z} \text { Moon }))
\end{aligned}
$$

where $\mathrm{D}$ is declination, $\mathrm{L}$ is known latitude of the observer, LHA is local hour angle based on assumed time and longitude and $\mathrm{Z}$ is the calculated azimuth of the body for these inputs. LD is Lunar Distance, HO Moon is the calculated altitude (HC) of the moon for the aforementioned inputs, corrected for parallax and refraction; HO Body is $\mathrm{HC}$ of the other body for the same, also corrected for parallax (where applicable - i.e. not in the case of stars) and refraction, and $\mathrm{Z}$ Body $-\mathrm{Z}$ Moon is the difference in calculated azimuths between the other body and the moon for that assumed time and position. Equations (1) and (2) must be 
carried out separately for the Moon and for the other body in order to supply the entering arguments for Equation (3).

The navigator then applies semi-diameter and augmentation corrections to that distance. The result is the distance as it would appear to an observer on Earth's surface at the assumed time and longitude.

It must be noted that in Brunner's (2005) method, a correction for IE of the sextant must be applied to the lunar distance actually observed. In the Stark method, the IE correction is factored in as part of the clearing process, but this is not so in Brunner's method.

At the end of each iteration of calculations, the difference in calculated lunar distance and observed lunar distance is converted from angular distance to time at a rate of 115 seconds of time to 1' of arc (Brunner, 2005. P.11). This time difference is then applied as a correction to the assumed time used at the beginning of that iteration, and, converted to longitude ( 1 ' of longitude being equal to $4 \mathrm{~s}$ of time), also applied as a correction to the corresponding assumed longitude (Brunner, 2005. P. 11). This results in a new assumed time and longitude with which to begin the next iteration. Calculated lunar distance should converge with observed lunar distance in successive iterations. True GMT and longitude of the observer (assuming negligible errors in observation, which is highly unlikely in practice) are found when calculated lunar distance and observed lunar distance agree.

\section{CONCLUSIONS.}

3.1. Stark and Brunner methods compared. Both of these methods are a good deal more laborious than the more commonplace procedures of celestial navigation. For navigators who wish to simply practice making the lunar distance observation itself in the most efficient way, it should be noted that software is available for smartphones, tablets, programmable calculators and personal computers which will perform all of the calculations in almost no time at all. Free web-based calculators are also available, but these require an Internet connection. Brunner also notes that a programmable calculator can be programmed to use his method, for those able to write their own algorithms and routines (Brunner, 2005).

Contemporaneously, one argument for maintaining celestial skills is the utility of celestial navigation as an emergency substitute for electronic navigation. For this purpose, the author prefers Stark's method to Brunner's, for ease of use and speed. In addition, it must be noted that during the process of working Brunner's method with a non-programmable scientific calculator, pencil, and paper, rounding errors and errors of transposition in the hundredths or thousandths places can be made. Such errors do not become apparent until the entire iteration of calculations is complete, at which point all of the math must be gone over carefully and all calculations reworked and corrected. From a practical standpoint, it seems unlikely that many watch-standers will be able to dedicate the time and attention necessary to prevent errors in successive iterations of calculations while also attending to the numerous other duties required of a deck officer. Errors may, of course, be made when working observations by Stark's method as well. However, with Stark's method, significant errors generally become apparent as soon as they are made and correcting them is simply a matter of changing one entry on the worksheets provided with Stark's Tables. Additionally, as all solutions in Stark's method are a matter of simple arithmetic, the entire process is streamlined and requires a good deal less time and dedicated concentration.

However, the Stark method depends on the observer's ability to take altitudes of both bodies being used, both before and after the observation for lunar distance. When working 
under an intermittently cloudy sky, or with a broken, difficult, or obscured horizon, the Stark method of clearing the distance cannot be used. In such instances Brunner's method is a good alternative. In this case the advantage in Brunner's method lies in the fact that it does not rely on observed altitudes to provide a solution, and indeed the method can be worked without taking altitudes of either body, provided the navigator has a dead-reckoning position and a very rough assumed time as a starting point.

3.2. Usefulness of lunar distance methods in general. The reliability and low cost of modern timepieces, and the redundancy of modern marine communications equipment makes it unlikely that time data would become suddenly unavailable. For practicing the technique of finding time by lunar distance as a hobby or for an appreciation of historical context, either Stark's or Brunner's methods will work well, although neither is an exact replication of the methods available to navigators in the eighteenth and nineteenth centuries. The strongest argument for making lunar distance observations is that it is perhaps the most difficult possible operation within the discipline of celestial navigation. A navigator who can master lunar distance observations should have no difficulties with the more commonly used celestial navigation techniques.

\section{ACKNOWLEDGEMENTS}

The author would like to thank Captain Chris Nolan of the Sea Education Association, Captains Andy Chase and Rick Miller of the Maine Maritime Academy and Professor Craig Marin of the Sea Education Association.

\section{REFERENCES}

Brunner, W. (2005). Longitude By The Method Of Lunar Distance. Resources, Starpath School of Navigation, LLC. www.starpath.com/resources2/brunner-lunars.pdf. Accessed 1 June 2017.

Lecky, S. T.S., and Allingham, W. (1918). Wrinkles in Practical Navigation. 19th ed. George Phillip \& Son.

Maskelyne, N. (1767). The Nautical Almanac and Astronomical Ephemeris For The Year 1767. Commissioners of Longitude.

National Geo-spatial Intelligence Agency. (2017a). The American Practical Navigator; An Epitome of Navigation, Vol. 1. U.S. National Geo-spatial Intelligence Agency.

National Geo-spatial Intelligence Agency. (2017b). The American Practical Navigator; An Epitome of Navigation, Vol. 2. U. S. National Geo-spatial Intelligence Agency.

Raper, H. (1908). The Practice of Navigation and Nautical Astronomy. 19th ed. J.D. Potter.

Sadler, D.H. (1978). Lunar Methods for 'Longitude Without Time. The Journal of Navigation, 31(2), 244-249.

Stark, B. (2010). Stark Tables for Clearing the Lunar Distance and Finding Universal Time by Sextant Observation. 3rd ed. Starpath Publications. 